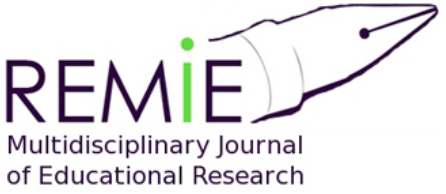

Multidisciplinary Journal of Educational Research

\section{Hipatia Press}

www.hipatiapress.com

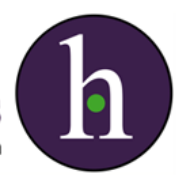

Instructions for authors, subscriptions and further details:

http://remie.hipatiapress.com

\title{
Latino Immigrant Youth and Interrupted Schooling: Dropouts, Dreamers and Alternative Pathways to College.
}

\section{Oriol Ríos}

1) Universidad Rovira i Virgili. España

Date of publication: June $15^{\text {th }}, 2016$

Edition period: June 2016 - October 2016

To cite this article: Ríos, O. (2016). Latino Immigrant Youth and Interrupted Schooling: Dropouts, Dreamers and Alternative Pathways to College [Review of the book]. REMIE-Multidisciplinary Journal of Educational Research, 6(2), 205-207. doi:10.17583/remie.2016.2068

To link this article: http://dx.doi.org/doi:10.17583/remie.2016.2068

\section{PLEASE SCROLL DOWN FOR ARTICLE}

The terms and conditions of use are related to the Open Journal System and to Creative Commons Attribution License (CC-BY). 
REMIE - Multidisciplinary Journal of Educational Research Vol. 6

No. 2 June 2016 pp. 205-207

\section{Review}

Lukes, M. (2015). Latino Immigrant Youth and Interrupted Schooling: Dropouts, Dreamers and Alternative Pathways to college. Houston, TX: Multilingual Matters.

"De verdad, no nos ven". Estas palabras, recogidas de uno de los jóvenes latinos participantes en la investigación desarrollada por Marguerite Lukes, ilustran de forma certera el principal cometido que movilizó a la autora a desarrollar la investigación que culmina en su obra Latino Immigrant Youth and Interrupted Schooling. Dropouts, Dreamers and Alternative: visibilizar la realidad de infinidad de personas jóvenes inmigrantes en Estados Unidos, procedentes de diferentes países de Centroamérica y Sudamérica, con trayectorias educativas heterogéneas, que participan activamente en el sistema educativo estadounidense a través de iniciativas de educación de personas adultas.

Lukes, investigadora con una extensa trayectoria en el ambito de la práctica educativa con diferentes poblaciones en contextos con diversidad linguística y cultural, arroja luz en su investigación sobre la realidad de un sector tradicionalmente invisibilizado por parte de las investigaciones que analizan el paso por el sistema educativo del alumnado de origen inmigrante en Estados Unidos. Si bien estas personas jóvenes de origen latino han sido tradicionalmente incluidas dentro del amplio paraguas de los drop out students (estudiantes que abandonan el sistema educativo), la autora desvela a través de su investigación, que estas personas jóvenes constituyen un grupo altamente heteregéneo, que no puede ser observado únicamente desde un enfoque reduccionista y estereotipado que les achaca una falta de interés o motivación por completar su educación. Muy al contrario, el libro recoge innumerables relatos e historias de vida que dan cuenta de los esfuerzos cotidianos de un amplio sector de la poblacion 
joven latina que, no habiendo concluido los estudios secundarios por los canales tradicionales, en gran medida como resultado de haber vivido procesos migratorios que les obligaron a interrumpir su trayectoria escolar, han retomado sus proyectos personales y educativos, a través de diferentes iniciativas y programas educativos, que combinan con frecuencia con experiencias profesionales.

Tres elementos convierten a esta obra en una atractiva y firmemente fundamentada aproximación a la realidad de este sector de la población. En primer lugar, cabe destacar la solidez con que la autora fundamenta su estudio en las contribuciones de algunos de los principales autores y autoras que desde diferentes disciplinas, como la Sociología o los estudios culturales, que han aportado análisis rigurosos de las desigualdades que afectan a los colectivos más vulnerables en el contexto norteamericano. A "hombros" de las aportaciones de Gary Orfield, Patricia Gándara, Alejandro Portes o Carola Suárez-Orozco, entre otros, la autora retrata los principales desafíos a los que debe hacer frente la población joven latina en Estados Unidos, así como los mecanismos que explican las persistentes desigualdades educativas que combaten día a día estos jóvenes en grandes contextos urbanos tales como la ciudad de Nueva York. En segundo lugar, es preciso resaltar la rigurosidad a la hora de construir un estudio de corte cuantitativo que a través de los datos desmiente algunos de los principales mitos que circulan en torno al paso por el sistema educativo de este sector de la población, como la baja importancia que otorgan a la educación. En tercer lugar, es preciso resaltar la forma en que la autora ha sido capaz de complementar este estudio cuantitativo con una profunda aproximación cualitativa, que se nutre de las voces de Víctor (Honduras), Elena (Ecuador), Ramón (México), Nelson (El Salvador) o Altagracia (República Dominicana), que con sus historias ofrecen un detallado testimonio de las luchas diarias por culminar sus expectativas y alcanzar sus sueños, transformando así su realidad por medio de la educación.

El resultado es un análisis mixto, fundamentado y éticamente responsable, que resulta de gran utilidad para comprender en profundidad los mecanismos de estratificación social y educativa que operan en las sociedades multiculturales, como es el caso de los Estados Unidos. De esta forma, el libro constituye una aportación original, que avanza en la 
REMIE-Multidisciplinary Journal of Educational Research, 6(1) 207 investigación existente sobre esta temática y supera algunos enfoques tradicionales, entre los que han predominado los análisis unidimensionales o monolíticos de la realidad educativa de la población joven latina en el contexto estadounidense.

Oriol Ríos, Universidad Rovira i Virgili joseoriol.rios@urv.cat 